\title{
Glossopharyngeal neuralgia associated with cardiac syncope: long term treatment with permanent pacing and carbamazepine
}

\author{
R Trevor Johnston, Vincent J Redding
}

\begin{abstract}
Glossopharyngeal neuralgia associated with cardiac syncope developed in a 53 year old man. Symptoms were controlled with temporary and permanent transvenous pacing and carbamazepine.
\end{abstract}

Glossopharyngeal neuralgia with syncope is a rare syndrome. It is characterised by paroxysms of unilateral intense lightning pain felt deep in the throat and pharynx, radiating to the ipsilateral ear, and associated with transient loss of consciousness caused by brief periods of asystole. Transient cerebral ischaemia may cause convulsions.

\section{Case report}

A 53 year old man was admitted in December 1976 with a two week history of recurrent syncopal attacks. He complained of a severe shooting pain in the left side of his throat radiating to the left ear, followed by dizziness and sometimes loss of consciousness lasting for a few seconds. These paroxysms had occurred several times a day and were precipitated by swallowing (mainly when drinking cold beer), chewing, brushing his teeth, and sneezing. Three weeks previously he had had non-specific "flu-like" symptoms with a productive cough, and was treated with ampicillin. He had been hypertensive for five years, and was treated with methyldopa and propranolol.

The patient was overweight and anxious. His oral hygiene was poor. His pulse was 76 per minute and regular. Blood pressure was $170 / 100 \mathrm{~mm} \mathrm{Hg}$. Auscultation of the heart and lungs was normal, there were no carotid bruits, and there was no abnormality of the central nervous system. Ocular fundi showed arteriovenous nipping.

During the initial clinical examination the patient had two brief attacks of pain with an intense grimace followed immediately by complete loss of consciousness. At this time the electrocardiographic monitor recorded episodes of sinus arrest with junctional escape beats (fig 1).

Glossopharyngeal neuralgia with cardiac syncope was diagnosed. Temporary bipolar ventricular pacing was established via the right subclavian vein. Over the next week the patient continued to have neuralgia but was protected from syncopal attacks by the demand pacemaker (fig 2).

Further examination of his throat showed congestion of the posterior pharyngeal wall with oedema of the uvula and tonsillar fauces. The left upper molars were loose and carious. During the dental examination the patient developed typical pain, followed by asystole which was interrupted by pacemaker intervention. A trigger zone was noted around the left peritonsillar and paramolar region, which when touched with cotton wool or ethyl chloride spray induced an attack. There was no evidence of hypersensitivity of the carotid sinus-carotid massage on either side did not produce significant bradycardia, hypotension, or asystole.' Somatic pain produced by squeezing the achilles tendon failed to induce an attack.

\footnotetext{
Department of Cardiology, The Regional Cardiothoracic Unit, Groby Road Hospital, Leicester R T Johnston V J Redding

Correspondence to Dr R Trevor Johnston Department of Cardiology, Regional Cardiothoracic Unit, Groby Road Hospital, Leicester LE3 9QE.
}
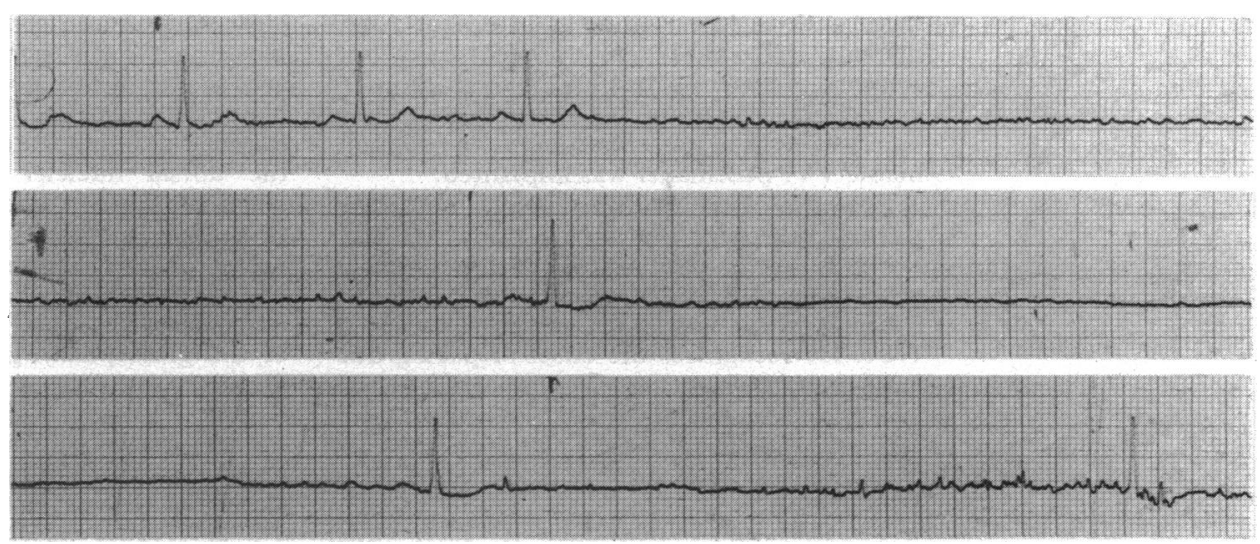

Figure 1 Continuous electrocardiographic recording (paper speed $25 \mathrm{~mm} / \mathrm{s}$ ) showing sinus arrest after pharyngeal pain. 

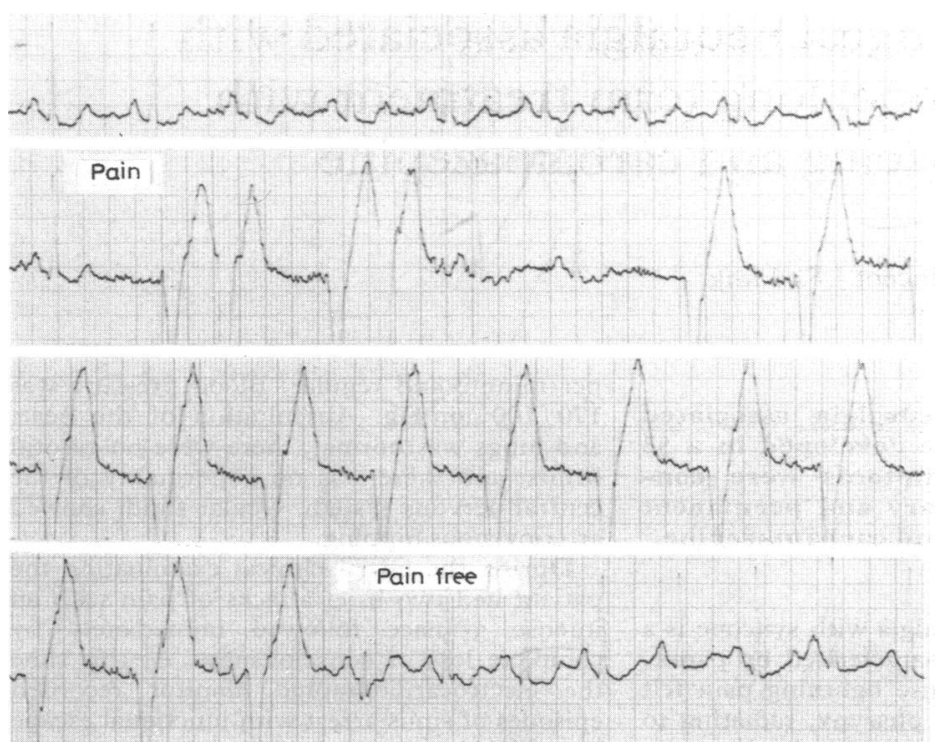

Figure 2 Continuous electrocardiographic recording (paper speed $25 \mathrm{~mm} / \mathrm{s}$ ) showing sinus rhythm preceding pharyngeal pain, and ventricular demand pacing during an episode of neuralgia. tumour affecting the cerebellopontine angle. ${ }^{2}$ In 1921 Harris reported the first idiopathic case associated with syncope, ${ }^{3}$ and Riley et al in 1942 reported two cases associated with syncope and seizures. ${ }^{4}$ Several other cases have been reported. Glossopharyngeal neuralgia may be associated with trigeminal or vagus neuralgia, ${ }^{56}$ hypersecretion of the ipsilateral parotid gland, ${ }^{7}$ cerebral symptoms such as loss of consciousness and convulsions in the presence of normal heart rate and blood pressure, ${ }^{8}$ elongation of the styloid process or calcified styloid ligament, ${ }^{9}$ intracranial tumours and vascular abnormalities, ${ }^{210}$ multiple sclerosis, ${ }^{11}$ Paget's disease and carcinomatosis affecting the base of the skull, ${ }^{12}$ and after dental extraction. ${ }^{13}$

Rushton et al in 1981 reviewed 217 patients with glossopharyngeal neuralgia seen at the Mayo Clinic; only four had associated syncope..$^{14}$ Lee et al in 1975 reviewed 17 cases of glossopharyngeal neuralgia associated with syncope. ${ }^{13}$ Most were in patients over the age of 50 years; there was no sex difference. Most patients did not seek medical help for neuralgia until they experienced syncopal attacks. The latent period from the onset of neuralgia to that of syncope varied from a few weeks to several years. The onset of the disease is abrupt and the pain is characteristically paroxysmal, unilateral, following the path of the glossopharyngeal nerve distribution, and affecting the left side more frequently than the right.

The presence of trigger zones in glossopharyngeal neuralgia is less common than in trigeminal neuralgia. They are generally situated in the peritonsillar fossa of the same side, as in our patient. Carotid sinus massage is said to be ineffective in glossopharyngeal neuralgia, which distinguishes it from the hypersensitive carotid sinus syndrome. ${ }^{1516}$ However, Jamshidi and Masroor and Alpert et al described two elderly women with glossopharyngeal neuralgia with asystole and a hypersensitive carotid sinus. ${ }^{1718}$

The mechanism and exact neural connections responsible for bradycardia after the neuralgic pain are still not completely understood. The most acceptable explanation is the close anatomical relation of the vagus and glossopharyngeal nerves. ${ }^{19}$. Sensory impulses arising from the posterior pharyngeal wall reach the tractus solitarius and its nucleus in the medulla oblongata and communicate with the dorsal nucleus of the vagus through collateral fibres. The dorsal nucleus is the principal autonomic nucleus supplying parasympathetic fibres to the heart, bronchi, and abdominal viscera. The carotid sinus nerve (nerve of Hering) is an afferent branch of the glossopharyngeal nerve and conducts impulses from the carotid body and sinus to the medulla and plays an important role in the regulation of the heart rate and blood pressure. Impulses arising from irritative or ischaemic lesions in the peripheral course of the glossopharyngeal nerve may reach the tractus solitarius and then be relayed to the dorsal nucleus of the vagus through 
collaterals, or there may be a functional spillover of impulses via artificial motor-tosensory synapses into the carotid sinus nerve as it travels with the glossopharyngeal nerve in the region of the jugular foramen. ${ }^{1618}$

It has been suggested that neuralgic pain only of extreme severity will activate the glossopharyngeal-vagal reflex arc resulting in bradycardia, hypotension, and syncope. This may be an explanation of disparities in the latent period between the onset of neuralgia and syncope seen in the various reported cases.

The basic aim of treatment should be control of pain, which will in turn prevent reflex bradycardia and syncope. This can be achieved either medically or surgically. Local nerve blocks and spraying of the pharyngeal mucosa with local anaesthetic have been used but give only temporary relief. ${ }^{16}$ In our patient local spray with ethyl chloride triggered an attack. In the chronic cases intracranial section of the glossopharyngeal nerve is a definitive and permanent treatment, but leaves irreversible and unwanted side effects. ${ }^{20}$ Surgical resection of an anomalous styloid process may be possible. ${ }^{9}$

Treatment with anticonvulsant drugs has sometimes been successful. Phenytoin gives variable results, ${ }^{714}$ but results with carbamazepine have been more encouraging, ${ }^{142122}$ though this drug may lose its effect with time. ${ }^{23}$ Atropine and isoprenaline have been used as temporary emergency measures to abolish bradycardia but they have no effect on pain.

Khero and Mullins in 1971 described a patient treated by temporary transvenous pacemaker while waiting for surgical section of the nerve. ${ }^{24}$ Jamshidi and Masroor in 1976 and Alpert et al in 1977 treated their patients with carbamazepine and permanent demand pacemakers because they also suffered from hypersensitive carotid sinus syndrome. ${ }^{17} 18 \mathrm{In}$ our patient the carotid sinus was not hypersensitive and long term treatment with a permanent pacemaker and carbamazepine while not completely abolishing neuralgic pain had an acceptable outcome.

Idiopathic glossopharyngeal neuralgia associated with cardiac syncope is usually a self limiting disease with no reported fatal outcome. Spontaneous recovery is not un- common but relapses may occur. We suggest that initially it should be treated with carbamazepine and a temporary demand pacemaker. If, however, the disorder is long standing, with a history of remissions and relapses, a permanent demand pacemaker combined with medical treatment should be considered as a more acceptable alternative to surgical section of the nerve.

1 Walter PK, Crawsley IS, Dorney ER. Carotid sinus hypersensitivity and syncope. Am J Cardiol 1978;42:396-403.

2 Weisenberg TH. Cerebellopontine tumour diagnosed for six years as tic Douloureux. The symptoms of irritation of ninth and twelfth cranial nerves. JAMA 1910;54:1600-4.

3 Harris $W$. Persistent pain in lesions of the peripheral and central nervous system. Br Med J 1921;ii:896-900.

4 Riley HA, German WJ, Wortis H, et al. Glossopharyngeal neuralgia initiating or associated with cardiac arrest. Trans Am Neurol Assoc 1942;68:28-9.

5 Chawla JC, Falconer MA. Glossopharyngeal and vagal neuralgia. Br Med J 1967;ili:529-31.

6 Brzustowicz RJ. Combined trigeminal and glossopharyngeal neuralgia. Neurology 1955;5:1-10.

7 Kjellin K, Muller R, Widen LS. Glossopharyngeal neuralgia associated with cardiac arrest and hypersecretion from gia associated with cardiac arrest and hypersecretion from

8 Thompson JL. Glossopharyngeal neuralgia accompanied by unconsciousness. $J$ Neurosurg 1954;11:511-4.

9 Graf CJ. Glossopharyngeal neuralgia and ossification of the stylohyoid ligament. J Neurosurg 1959;16:448-53.

10 Lillie HI, Craig WMcK. Anomalous vascular lesion in cerebellopontine angle, severe neuralgia pain in the ear and profound nervous disturbance: operation and recovery. Arch Otolaryngol 1936;23:642-5.

11 Kahana E, Leibowitz U, Alter M. Brainstem and cranial nerve involvement in multiple sclerosis. Acta Neurol Scand 1973;49:269-79.

12 Orton CI. Glossopharyngeal neuralgia: its diagnosis and treatment. Br J Oral Surg 1972;9:228-32.

13 Lee YT, Lee TK, Tsai HC. Glossopharyngeal neuralgia as a cause of cardiac syncope. (A case report with review of cause of cardiac syncope. (A case report with
literature.) J Form Med Assoc 1975;74:103-7.

14 Rushton JG, Stevens JC, Miller RH. Glossopharyngeal (vago-glossopharyngeal) neuralgia. A study of 217 cases. Arch Neurol 1981;38:201-5.

15 Garretson HD, Elvidge AR. Glossopharyngeal neuralgia with asystole and seizures. Arch Neurol 1963:8:26-31.

16 Kong Y, Heyman A, Entman ML, et al. Glossopharyngeal neuralgia associated with bradycardia, syncope and seizures. Circulation 1964;30:109-13.

17 Jamshidi A, Masroor MA. Glossopharyngeal neuralgia with cardiac syncope. Treatment with a permanent cardiac pacemaker and carbamazepine. Arch Intern Med 1976; 136:843-5.

18 Alpert JN, Armbrust CA, Akhavi M, Stamatiou ES, Killian JM, De Shazo M. Glossopharyngeal neuralgia, asystole, and seizures. Arch Neurol 1977;34:233-5.

19 Karnosh LJ, Gardner WJ, Stowell A. Glossopharyngeal neuralgia. Physiological consideration of the role of ninth neuralgia. Physiological consideration of the role of ninth and tenth cranial nerves.

20 Ray BS, Stewart HJ. Glossopharyngeal neuralgia. A cause of cardiac arrest. Am Heart J 1948;35:458-62.

21 Ekbom KA, Westerberg CE. Carbamazepine in glossopharyngeal neuralgia. Arch Neurol 1966;14:595-6.

22 Saviolo R, Fiasconaro G. Treatment of glossopharyngeal neuralgia by carbamazepine. Br Heart J 1987;58:291-2.

23 Russell Rees J, Bicknell PG. Glossopharyngeal neuralgia with syncope. Br Med J 1979;i:754.

24 Khero BA, Mullins CB. Cardiac syncope due to glossopharyngeal neuralgia. Arch Intern Med 1971;128:806-8. 